\title{
BRANCHING PROCESSES IN SIMPLE RANDOM WALK
}

\author{
MEYER DWASS
}

ABSTRACT. Let $N(a)$ be the number of overcrossings of height $a$ in a simple random walk. For $p<1 / 2$, the process $N(0), N(1), \ldots$ is a branching process which eventually becomes extinct. For $1 / 2<$ $p, N(0), N(1), \ldots$ is a stationary process which is a branching process with immigration.

1. Introduction. We show that a certain branching process and a branching process with immigration arise in simple random walk when $p \neq 1 / 2$. By simple random walk we mean the sequence of random variables, $S_{0}, S_{1}, \ldots$, $S_{0}=0, S_{n}=X_{1}+\cdots+X_{n^{\prime}} n=1,2, \ldots$, where the $X_{i}^{\prime}$ 's are independent and identically distributed;

$$
X_{i}=\left\{\begin{aligned}
1 & \text { with probability } p, \\
-1 & \text { with probability } 1-p=q .
\end{aligned}\right.
$$

Definition 1. An overcrossing of height $a$ takes place at time $n$ if $S_{n}=a, S_{n-1}=a+1, S_{i}=a$ for some $i<n$.

Definition 2. $N(a)$ denotes the total number of overcrossings of height a. $(N(a)$ is finite if $p \neq 1 / 2$.)

\section{Statement of theorems.}

Theorem 1。 For $p<1 / 2, N(0), N(1), \ldots$ evolves as a branching process with

$$
\begin{aligned}
E t^{N(0)} & =(1-p / q) /(1-p t / q), \\
E\left(t^{N(a+1)} \mid N(a)=k\right) & =[q /(1-p t)]^{k}, \quad a, k=0,1, \ldots
\end{aligned}
$$

(In other words, each of the elements of the preceding generation independently gives rise to a random number of new elements, according to the progeny generating function $q /(1-p t)$.)

Theorem 2. For $1 / 2<p, N(0), N(1), \ldots$ evolves as a branching process,

Received June 17, 1974.

AMS (MOS) subject classifications (1970). Primary 13J15, $13 \mathrm{~J} 80$.

Key words and phrases. Branching process, branching process with immigration, random walk, simple random walk.

1 Without further notice, the dummy variables in all generating functions are assumed to be less than 1 in absolute value. 
with immigration, with

$$
\begin{gathered}
E t^{N(0)}=(1-q / p) /(1-q t / p), \\
E\left(t^{N(a+1)} \mid N(a)=k\right)=[p /(1-q t)]^{k}[p /(1-q t)], \quad a, k=0,1, \ldots .
\end{gathered}
$$

(In other words, each of the elements of the preceding generation inde. pendently gives rise to a random number of new elements, according to the progeny generating function $p /(1-q t)$; in addition, in each generation there is an influx of a random number of new individuals by immigration according to the same generating function $p /(1-q t)$.

3. Proofs。The proofs of Theorems 1 and 2 will proceed as follows.

Step 1. Define

$T=$ time at which first overcrossing of 0 takes place. (For $p \neq 1 / 2$, $P(T<\infty)<1$.

$V(a)=$ total number of overcrossings of $a$ up to time $T$.

$R_{n}\left(i_{1}, \ldots, i_{n}\right)=$ probability of all paths which start at height 1 , overcross height $1 i_{1}$ times, overcross height $2 i_{2}$ times,..., overcross height $n i_{n}$ times; do not overcross height 0 enroute, and end up eventually at height 1 .

$V_{n}\left(s_{1}, \ldots, s_{n}\right)=E\left(s_{1}^{V(1)} \ldots \ldots s_{n}^{V(n)} \mid T<\infty\right)$.

Step 2.

$$
P(T<\infty)= \begin{cases}p / q, & p \leq 1 / 2 \\ q / p, & 1 / 2 \leq p\end{cases}
$$

$$
E\left(t^{V(1)} \mid T<\infty\right)= \begin{cases}q /(1-p t), & p \leq 1 / 2 \\ p /(1-q t), & 1 / 2 \leq p\end{cases}
$$

Proof. (a) is a standard fact about simple random walk. Part (b) follows from (a) by the computation,

$$
P(V(1)=k, T<\infty)= \begin{cases}(p / q)(p \cdot 1)^{k} q=p^{k+1}, & p \leq 1 / 2, \\ 1 \cdot(p \cdot q / p)^{k} q=q^{k+1}, & 1 / 2 \leq p .\end{cases}
$$

Step 3.

$$
\begin{aligned}
E\left(s_{2}^{V(2)} \ldots \ldots s_{n}^{V(n)} \mid V(1)=k, T<\infty\right)=\left[V_{n-1}\left(s_{2}, \ldots, s_{n}\right)\right]^{k}, & \\
& k=0,1, \ldots .
\end{aligned}
$$

Proof. If $k=0$, then $V(2)=\cdots=V(n)=0$ and the assertion holds, so assume now that $1 \leq k$. Suppose that $p \leq 1 / 2$. From the definition of $R_{n}$, 


$$
P\left(V(1)=i_{1}, \ldots, V(n)=i_{n}, T<\infty\right)=(p / q) R_{n}\left(i_{1}, \ldots, i_{n}\right) q .
$$

For $1 \leq k$, we also have

$$
\begin{aligned}
P(V(1)=k, & \left.V(2)=i_{2}, \ldots, V(n)=i_{n}, T<\infty\right) \\
& =\sum(p / q)\left[p R_{n-1}\left(j_{12}, \ldots, j_{1 n}\right) q\right] \cdots\left[p R_{n-1}\left(j_{k 2}, \ldots, j_{k n}\right) q\right] q
\end{aligned}
$$

where the summation is over all vector sums of $k(n-1)$-tuples such that

$$
\left(j_{12}, \ldots, j_{1 n}\right)+\cdots+\left(j_{k 2}, \ldots, j_{k n}\right)=\left(i_{2}, \ldots, i_{n}\right) .
$$

Hence, computing generating functions, we have

$$
\begin{aligned}
E\left(s_{1}^{V(1)}\right. & \left.\cdots s_{n}^{V(n)} ; V(1)=k, T<\infty\right) \\
& =(p / q)\left[q E\left(s_{2}^{V(1)} \cdots \cdots s_{n}^{V(n-1)} ; T<\infty\right)\right]^{k} q
\end{aligned}
$$

from which the result follows by dividing through by $P(V(1)=k, T<\infty)=$ $p^{k+1}$. The proof for $1 / 2 \leq p$ is similar and is left to the reader.

Step 4. $V_{n}\left(s_{1}, \ldots, s_{n}\right)=V_{1}\left(s_{1} V_{n-1}\left(s_{2}, \ldots, s_{n}\right)\right)$.

Proof.

$$
\begin{aligned}
& E\left(s_{1}^{V(1)} \ldots \cdots s_{n}^{V(n)} \mid T<\infty\right) \\
& \quad=\sum_{k} E\left(s_{1}^{V(1)} \ldots \cdots s_{n}^{V(n)} \mid V(1)=k, T<\infty\right) P\left(V(1)^{\cdot}=k \mid T<\infty\right) \\
& \quad=\sum_{k} s_{1}^{k}\left[V_{n-1}\left(s_{2}, \ldots, s_{n}\right)\right]^{k} P(V(1)=k \mid T<\infty) \quad \text { (by Step 3). }
\end{aligned}
$$

This last expression equals the right side of the assertion.

Step 5. Let $Y(0), Y(1), Y(2), \ldots$ be a branching process with $Y(0)=1$ and $E\left(t^{Y(n+1)} \mid Y(n)=k\right)=F^{k}(t)$.

Define the joint generating function of $Y(1), \ldots, Y(n)$ to be

$$
W_{n}\left(s_{1}, \ldots, s_{n}\right)=E\left(s_{1}^{Y(1)} \ldots s_{n}^{Y(n)}\right) .
$$

Then,

$$
W_{n}\left(s_{1}, \ldots, s_{n}\right)=F\left(s_{1} W_{n-1}\left(s_{2}, \ldots, s_{n}\right)\right) .
$$

The joint distribution of $Y(1), \ldots, Y(n)$ is uniquely determined by this iterative relationship. 
Proof。

$$
\begin{aligned}
& E\left(s_{1}^{Y(1)} \ldots s_{n}^{Y(n)}\right)=\sum_{k} E\left(s_{1}^{Y(1)} \ldots \ldots s_{n}^{Y(n)} \mid Y(1)=k\right) P(Y(1)=k) \\
& \quad=\sum_{k} s_{1}^{k}\left[W_{n-1}\left(s_{2}, \ldots, s_{n}\right)\right]^{k} P(Y(1)=k)=F\left(s_{1} W_{n-1}\left(s_{2}, \ldots, s_{n}\right)\right) .
\end{aligned}
$$

Step 6. Under the condition that $T<\infty$, the process $V(1), V(2), \ldots$ coincides in distribution with a branching process $Y(1), Y(2), \ldots$, where $Y(0)=1$ and

$$
E\left(t^{Y(n+1)} \mid Y(n)=k\right)= \begin{cases}{[q /(1-p t)]^{k},} & p \leq 1 / 2, \\ {[p /(1-p t)]^{k},} & 1 / 2 \leq p, \quad n, k=0,1, \ldots .\end{cases}
$$

Proof. It follows from Steps 4 and 5 and 2(b) that

$$
E\left(s_{1}^{V(1)} \ldots s_{n}^{V(n)} \mid T<\infty\right)=E\left(s_{1}^{Y(1)} \ldots s_{n}^{Y(n)}\right)
$$

for all $n=1,2, \ldots$.

Completion of proof of Theorem 1. Suppose that $p<1 / 2$. If $N(0)=0$ then automatically $N(1)=N(2)=\cdots=0$. Suppose that $N(0)=k>0$. Define $\left(V_{i}(1), V_{i}(2), \ldots, V_{i}(n)\right)=V_{i}$ to be the number of overcrossings of heights $1,2, \ldots, n$ between the time of the $(i-1)_{s t}$ and $i$ th overcrossing of height 0. $\left(V_{1}(a)\right.$ is the same as $V(a)$ as defined earlier. $)$ Then:

(a) the random vectors $V_{1}, V_{2}, \ldots$ are independent and identically distributed in the sense that

$$
\begin{aligned}
& P\left(V_{1}=v_{1}, \ldots, V_{k}=v_{k} \mid N(0)=k\right) \\
& \quad=P\left(V_{1}=v_{1} \mid T<\infty\right) \ldots P\left(V_{k}=v_{k} \mid T<\infty\right),
\end{aligned}
$$

$P\left(V_{i}=v\right)$ does not depend on $i$.

(b) $N(a)=V_{1}(a)+\cdots+V_{N(0)}$ if $N(0)$ is positive.

(c) $P(N(0)=k)=(p / q)^{k}(1-p / q), k=0,1, \ldots$.

By Step 6, the proof of the theorem is now complete.

Completion of proof of Theorem 2. For $1 / 2<p$, it is no longer true that if $N(0)=0$ that $N(1), N(2), \ldots$ are also zero. We must now be concerned with the overcrossings of height $a+1$ after the last overcrossing of height a. The number of such overcrossings plays the role of the immigration into the population at each generation. An easy computation shows that $P(N(1)$ $\left.=k \mid N(0)=0, S_{0}=0\right)=p q^{k}, k=0,1, \ldots$. For the rest, the proof is similar to that of Theorem 1 and we leave the details to the reader. 
4. Complements. Suppose that $p<1 / 2$. A direct calculation shows that

$$
P(N(a)=k)= \begin{cases}1-(p / q)^{a+1}, \\ (p / q)^{a}(p / q)^{k}(1-p / q), & 0<k .\end{cases}
$$

Hence,

$$
G_{a}(t)=E t^{N(a)}=1-(p / q)^{a+1}(1-t) /(1-p t / q) .
$$

Let $F(t)$ denote the progeny generating function, $F(t)=q /(1-p t)$. Since $N(a)$ evolves as a branching process, we must have that

$$
G_{a}(t)=G_{0}\left(F^{(a)}(t)\right), \quad a=1,2, \ldots,
$$

where $F^{(a)}$ is the $a$-fold iteration given by $F^{(a)}(t)=F\left(F^{(a-1)}(t)\right), a=2,3, \ldots$ It is easy to verify directly by induction that (4.1) satisfies (4.2)。

For $1 / 2<p$ we have that

$$
E t^{N(a)}=(1-q / p) /(1-q t / p)=G(t), \quad a=1,2, \ldots .
$$

(Since $N(a)$ is a Markov chain it follows that it is a strictly stationary process.) Since $N(0), N(1), \ldots$ evolves as a branching process with immigration, we must have that

$$
G(t)=G(F(t)) p /(1-q t)
$$

reflecting the relationship between $N(a+1)$ and $N(a)$ stated in Theorem 2, with $F(t)=p /(1-q t)$. It is easy to verify that (4.3) holds directly.

For $p<1 / 2, N(a)$ must equal 0 for suff iciently large $a$. This is consistent with the fact that

$$
\frac{d}{d t} q /\left.(1-p t)\right|_{t=1}=p / q<1
$$

In other words, if the expected number of progeny is less than 1, the branching process becomes extinct with probability 1.

5. References. The elementary facts that are needed about random walk and about branching processes can be found in [1].

\section{REFERENCES}

1. W. Feller, An introduction to probability theory and its applications. Vol. I, 3rd ed., Wiley, New York, 1968. MR 37 \#3604. 